\title{
Occupancy distributions of homogeneous queueing systems under opportunistic scheduling*
}

\author{
Murat Alanyali and Maxim Dashouk \\ Department of Electrical and Computer Engineering \\ Boston University
}

\begin{abstract}
We analyze opportunistic schemes for transmission scheduling from one of $n$ homogeneous queues whose channel states fluctuate independently. Considered schemes consist of the LCQ policy, which transmits from a longest connected queue in the entire system, and its low-complexity variants that transmit from a longest queue within a randomly chosen subset of connected queues. A Markovian model is studied where mean packet transmission time is $n^{-1}$ and packet arrival rate is $\lambda<1$ per queue. Transient and equilibrium distributions of queue occupancies are obtained in the limit as the system size $n$ tends to infinity.
\end{abstract}

*An earlier version of this manuscript appeared at the Information Theory and Applications Workshop, UCSD, 2008. 


\section{Introduction}

We analyze a queueing system that arises under opportunistic scheduling of packet transmissions from a collection of queues with time-varying service rates. The system of interest is motivated by cellular data communications in which a single transceiver serves multiple mobile stations through distinct channels. Transmission scheduling has been well-studied in this context under the guiding principle of opportunism, which broadly refers to exploiting channel variations to maximize transmission capacity in the long term. In this we paper consider two generic opportunistic scheduling policies and obtain asymptotically exact descriptions of the resulting queue length distributions in symmetric systems of statistically identical queues.

Explicit analysis of queue lengths under opportunistic scheduling is generally difficult due to model complexities and lack of closed-form expressions. In related work Tassiulas and Ephremides 10, considered a queueing system under an on/off channel model in which each queue is independently either connected, and in turn it is eligible for service at a standard rate, or disconnected and it cannot be serviced. It is shown that transmitting from a longest connected queue stabilizes queue lengths if that is at all feasible, and that it minimizes occupancy of symmetric systems in which queues have identical load and channel statistics. This policy is coined $L C Q$. Explicit description of queue length distributions under LCQ is not available, but several bounds for mean packet delay are obtained in [3, 6] for LCQ and some of its variants. In more general models that admit multiple transmission rates and simultaneous transmissions, max-weight scheduling policies and their variations are shown in [9, 7] to asymptotically minimize a range of occupancy measures along a certain heavy-traffic limit. In the special case when one queue can transmit at a time, max-weight transmits from a queue that maximizes the product of instantaneous queue length and transmission rate. Tails of queue length distributions under such policies are studied in [8, 12] via large deviations analysis.

Here we consider a system of $n$ queues under an on/off channel model in which each queue is connected independently with probability $q \in(0,1]$. A continuous-time Markovian model is adopted where packet transmission rate is $n$ and packet arrival rate is $\lambda<1$ per queue. It can be seen that $\lambda$ is also the load factor of the system; hence the condition $\lambda<1$ is necessary to have positiverecurrent queue lengths. We analyze this system for large values of the system size $n$, under the LCQ scheduling policy and under its low-complexity variant, namely $L C Q(d)$, that transmits from 
a longest queue within $d \geq 1$ randomly selected connected queues. It is apparent that $\mathrm{LCQ}(d)$ is not particularly suitable for non-symmetric systems, yet our goal here is to obtain a generic evaluation of its underlying principle, which may be tailored to specific circumstances.

We establish that as the system size $n$ increases equilibrium distribution of queue occupancies under the LCQ policy converges to the deterministic distribution centered at 0 . Hence asymptotically almost all queues are empty in equilibrium. The number of queues with one packet is $\Theta(1)$ and the number of queues with more than one packet is $o(1)$ as $n \rightarrow \infty$. In particular maximum queue size tends to one. The total number of packets in the system is therefore given by the number of nonempty queues, and this number is shown to have the same equilibrium distribution as the positive-recurrent birth-death process with birth rate $\lambda$ and death rate $1-(1-q)^{j}$ at state $j \in \mathbb{Z}_{+}$. Note that the latter rate is equal to the probability of having at least one connected queue within a given set of $j$ queues, and that the nature of the total system occupancy may be anticipated once maximum queue size is determined to be one. The obtained description leads to asymptotic mean packet delay via Little's law as the rate of packet arrivals to the system is readily seen to be $n \lambda$.

The analysis technique applied to LCQ can be extended, although with excessive tediousness, to symmetric max-weight policies in cases when each queue can be serviced independently at rate $n R$ for some random variable $R$. Above conclusions about LCQ offer substantial insight about queue occupancies in that more general setting. Namely if $R$ exceeds $\lambda$ with positive probability (note that this condition is necessary for positive recurrence of queue lengths), then stochastic coupling with a related LCQ system yields that the maximum queue length in equilibrium tends to 1 as $n \rightarrow \infty$. In turn, equilibrium distribution of total system occupancy should be expected to resemble that of a birth-death process with birth rate $\lambda$ and death rate $E\left[\max \left\{R_{1}, R_{2}, \cdots, R_{j}\right\}\right]$ at state $j$, where $R_{1}, R_{2}, \cdots, R_{j}$ are independent copies of $R$.

We obtain the equilibrium distribution $\left\{p_{k}\right\}_{k=0}^{\infty}$ of individual queue occupancy under the LCQ $(d)$ policy in the limit as $n \rightarrow \infty$. Specifically $p_{k}=v_{k}^{*}-v_{k+1}^{*}$ where $v_{0}^{*}=1$ and

$$
v_{k}^{*}=1-\sqrt[d]{1-\lambda v_{k-1}^{*}}, \quad k=1,2, \cdots
$$

This distribution is shown to have tails that decay as $\Theta\left((\lambda / d)^{k}\right)$ as queue size $k \rightarrow \infty$. Hence, in terms of tail occupancy probabilities, the choice parameter $d$ has the equivalent effect of reducing the system load by the same factor. Yet, for any fixed $d$, system occupancy under LCQ $(d)$ is larger 


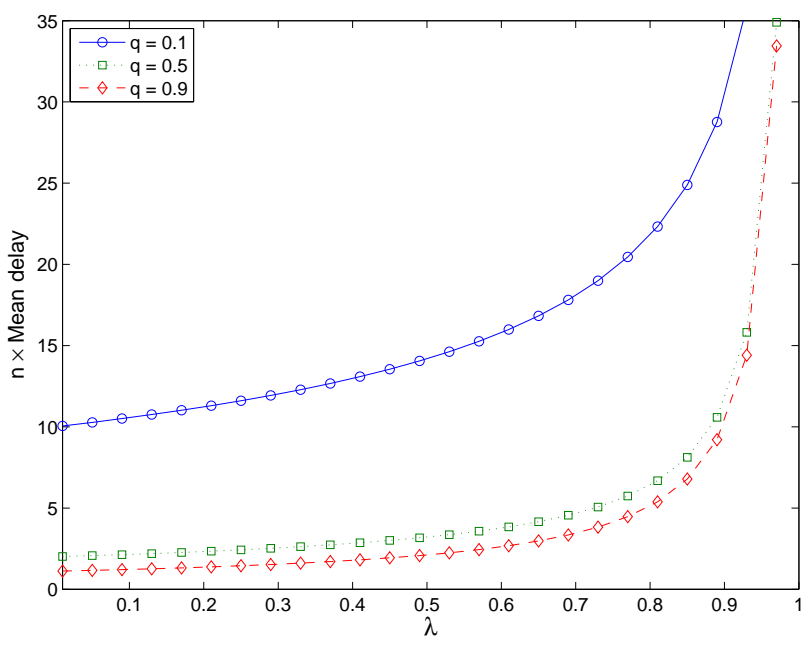

(a) LCQ

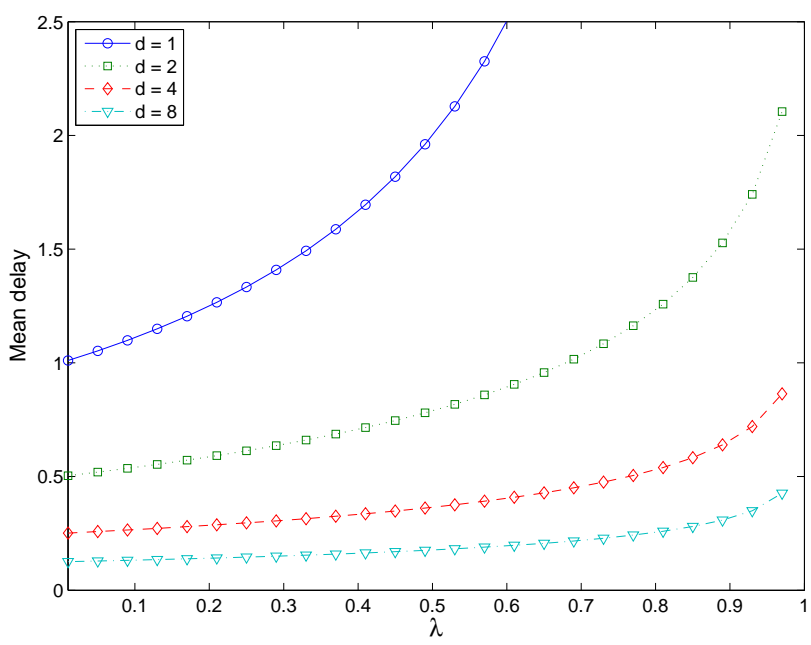

(b) $\operatorname{LCQ}(d)$

Figure 1: Mean packet delays as $n \rightarrow \infty$. Note the normalization in (a).

than that of LCQ by a factor of order $n$. Numerical values of asymptotic mean packet delay under LCQ and LCQ $(d)$ are illustrated in Figure 1. We also conclude that if $d$ is allowed to depend on $n$ so that $d \rightarrow \infty$ but $d / n \rightarrow 0$ then order of the alluded disparity reduces to $n / d$. This suggests that for moderate values of $d$ and $n \mathrm{LCQ}(d)$ and LCQ may be expected to have comparable packet delay.

The present analysis is based on approximating system dynamics via asymptotically exact differential representations that amount to functional laws of large numbers. Hence besides the mentioned equilibrium properties the paper also describes transient behavior of queue occupancies. The present analysis of LCQ $(d)$ is inspired by the work of Vvedenskaya et al. [11] which concerns an analogue of this policy that arises in routing and load balancing. It should perhaps be noted that the choice parameter $d$ appears to have a substantially more pronounced effect in the routing context. Our conclusions about the LCQ policy require a more elaborate technical approach. Here we apply a technique due to Kurtz [5] to obtain a suitable asymptotic description of the system. Related applications of this technique can be found in [1, 4, 13].

The rest of this paper is organized as follows. We continue in Section 2 with formal description of the model and the notation adopted in the paper. The policies LCQ $(d)$ and LCQ are analyzed respectively in Sections 3 and 4 . The paper concludes with final remarks in Section 5 . 


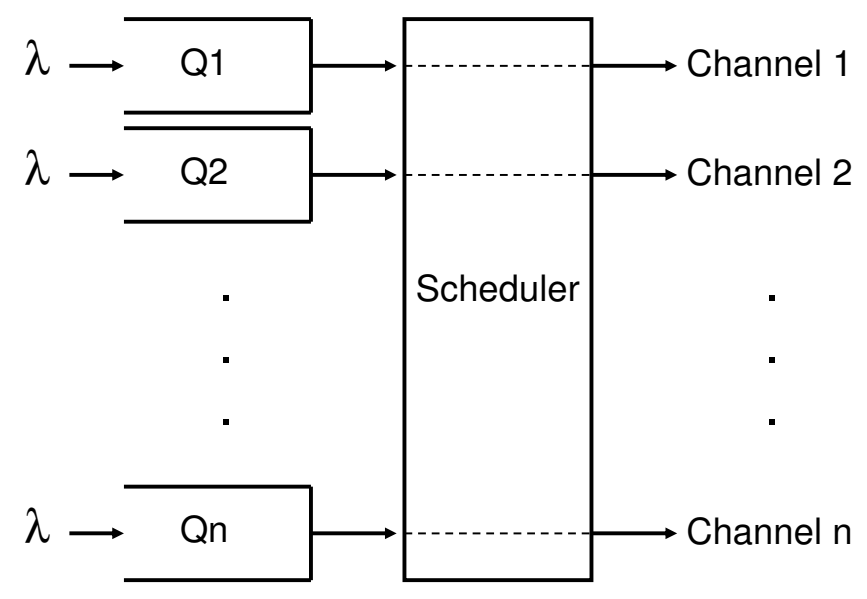

Figure 2: Sketch of the considered queueing system. At most one queue is serviced at a time, and each queue $i$ can be serviced only when channel $i$ is eligible for transmission.

\section{Queueing Model}

Consider $n$ queues each serving a dedicated stream of packet arrivals as illustrated in Figure 2 , Arrivals of each stream occur according to an independent Poisson process with rate $\lambda<1$ packets per unit time and transmission time of each packet is exponentially distributed with mean $n^{-1}$, chosen independently of the prior history of the system. Each queue is serviced by a designated channel but at most one channel can transmit at a time. Channel states fluctuate randomly and each channel is eligible for transmission with probability $q \in(0,1]$ independently of other channels. Queues with eligible channels are called connected. We assume that channel states remain constant during packet transmission and that they are determined anew, independently of each other and of the current queue lengths, just before the next transmission decision.

Let $m_{k}(t)$ denote the number of queues with $k$ or more packets at time $t$, and let

$$
u_{k}(t)=\frac{m_{k}(t)}{n}, \quad k=0,1,2, \cdots
$$

be the fraction of such queues in the system. In particular

$$
1=u_{0}(t) \geq u_{1}(t) \geq \cdots \geq 0
$$

the sequence $\left\{1-u_{k}(t)\right\}_{k=0}^{\infty}$ is the empirical cumulative distribution function of queue occupancies, and $\sum_{k \geq 1} u_{k}(t)$ is the empirical average queue occupancy in the system at time $t$. We denote by 
$P_{n}$ and $E_{n}$ respectively probabilities and expectations associated with system size $n$. In particular if $q_{i}(t)$ denotes the occupancy of the $i$-th queue at time $t$ then by the symmetry of the model

$$
E_{n}\left[u_{k}(t)\right]=P_{n}\left(q_{i}(t) \geq k\right)
$$

Let $U$ denote the collection of sequences $\mathbf{u}=\left\{u_{k}\right\}_{k=0}^{\infty}$ that satisfy relation (11), and endow $U$ with metric $\rho$ that is defined by

$$
\rho\left(\mathbf{u}, \mathbf{u}^{\prime}\right)=\sup _{k>0} \frac{\left|u_{k}-u_{k}^{\prime}\right|}{k}, \quad \mathbf{u}, \mathbf{u}^{\prime} \in U
$$

Note that convergence in $U$ is equivalent to coordinate-wise convergence, and that $U$ is compact as each coordinate lies in a compact interval.

For each time $t$ let $\mathbf{u}(t)$ denote the sequence $\left\{u_{k}(t)\right\}_{k=0}^{\infty}$. We represent the trajectory $(\mathbf{u}(t): t \geq 0)$ by the symbol $\mathbf{u}(\cdot)$, and say that $\mathbf{u}(\cdot)$ converges to a given trajectory $\mathbf{v}(\cdot)$ uniformly on compact timesets (uoc) if for all $t>0$

$$
\sup _{0 \leq s \leq t} \rho(\mathbf{u}(s), \mathbf{v}(s)) \rightarrow 0 \quad \text { a.s. as } n \rightarrow \infty \text {. }
$$

\section{$3 \mathbf{L C Q}(d)$}

We focus on $\operatorname{LCQ}(d)$ which randomly and independently selects $d$ connected queues and transmits from a longest queue within this collection. For convenience of analysis we assume that repetitions are allowed in the selection procedure, and that if all selected queues are empty or no connected queue exists at a scheduling instant then the scheduler makes a new selection after idling for the transmission time of a hypothetical packet. This latter assumption can be seen to imply that scheduling instances form a Poisson process of rate $n$.

For $k=1,2,3, \cdots$ let $\mathbf{e}_{k}=\left\{e_{k}(i)\right\}_{i=0}^{\infty}$ where

$$
e_{k}(i)=1\{i=k\}
$$

Here and in the rest of the paper $1\{\cdot\}$ denotes 1 if its argument is true and 0 otherwise. Jumps of the process $\mathbf{u}(\cdot)$ are of the form $\pm n^{-1} \mathbf{e}_{k}$ for some $k$. Namely $\mathbf{u}(\cdot)$ changes by $+n^{-1} \mathbf{e}_{k}$ whenever some queue with exactly $k-1$ packets has a new arrival, and by $-n^{-1} \mathbf{e}_{k}$ whenever a packet transmission is scheduled from a queue with exactly $k$ packets. The number of queues with $k-1$ packets at time $t$ is 
given by $n\left(u_{k-1}(t)-u_{k}(t)\right)$; hence the former event occurs at instantaneous rate $n \lambda\left(u_{k-1}(t)-u_{k}(t)\right)$. The latter event occurs if and only if, upon completion of a packet transmission, $(i)$ there exists a connected queue and $(i i)$ the scheduler inspects at least one connected queue with $k$ packets but none with more than $k$ packets. To determine the instantaneous rate of this event let $\tau$ be a scheduling instant and let

$$
\alpha_{n}=1-(1-q)^{n}
$$

Namely $\alpha_{n}$ is the probability that there exists a connected queue at time $\tau$. Since channel states are assigned independently of queue lengths at time $\tau$, a connected queue at this time has strictly less than $k$ packets with probability $1-u_{k}(\tau)$. Therefore, given that a connected queue exists, the maximum queue length inspected by the scheduler is equal to $k$ with (conditional) probability $\left(1-u_{k+1}(\tau)\right)^{d}-\left(1-u_{k}(\tau)\right)^{d}$. Since scheduling instants occur at constant rate $n$, instantaneous rate of transmissions from a queue of size $k$ is $n \alpha_{n}\left(\left(1-u_{k+1}(t)\right)^{d}-\left(1-u_{k}(t)\right)^{d}\right)$. In particular $\mathbf{u}(\cdot)$ is a time-homogeneous Markov process whose generator can be sketched as

$$
\mathbf{u} \leftarrow\left\{\begin{array}{lll}
\mathbf{u}+n^{-1} \mathbf{e}_{k} & \text { at rate } & n \lambda\left(u_{k-1}-u_{k}\right) \\
\mathbf{u}-n^{-1} \mathbf{e}_{k} & \text { at rate } & n \alpha_{n}\left(\left(1-u_{k+1}\right)^{d}-\left(1-u_{k}\right)^{d}\right),
\end{array} \quad k=1,2 \cdots\right.
$$

It offers some convenience in the subsequent discussion to represent the process $\mathbf{u}(\cdot)$ via the "random time change" construction of [2, Chapter 6]. Namely

$$
\begin{aligned}
\mathbf{u}(t)=\mathbf{u}(0)+\sum_{k=1}^{\infty} n^{-1} \mathbf{e}_{k} A_{k-1}\left(n \lambda \int_{0}^{t} u_{k-1}(s)-u_{k}(s) d s\right) \\
-\sum_{k=1}^{\infty} n^{-1} \mathbf{e}_{k} D_{k}\left(n \alpha_{n} \int_{0}^{t}\left(1-u_{k+1}(s)\right)^{d}-\left(1-u_{k}(s)\right)^{d} d s\right)
\end{aligned}
$$

where $A_{k-1}(\cdot), D_{k}(\cdot), k=1,2, \cdots$, are mutually independent Poisson processes each with unit rate. In informal terms, the processes $A_{k}(\cdot)$ and $D_{k}(\cdot)$ clock respectively arrivals to and departures from some queue with length $k$, and the construction (3) is based on suitably expediting these processes to match the instantaneous transition rates given in (2). Martingale decomposition of the Poisson processes used in (3) yields

$$
\mathbf{u}(t)=\mathbf{u}(0)+\sum_{k=1}^{\infty} \mathbf{e}_{k} \int_{0}^{t}\left(\lambda\left(u_{k-1}(s)-u_{k}(s)\right) d s-\alpha_{n}\left(\left(1-u_{k+1}(s)\right)^{d}-\left(1-u_{k}(s)\right)^{d}\right)\right) d s+\varepsilon(t)
$$

where $\varepsilon(t)=\left\{\varepsilon_{k}(t)\right\}_{k=0}^{\infty}$ is such that each coordinate process $\varepsilon_{k}(\cdot)$ is a real-valued martingale adapted to the filtration generated by $\mathbf{u}(\cdot)$. 
Theorem 3.1 Every subsequence of $\{n\}$ has a further subsequence along which $\mathbf{u}(\cdot)$ converges in distribution to a differentiable process $\mathbf{v}(\cdot)$ such that $v_{0}(t) \equiv 1$ and

$$
\frac{d}{d t} v_{k}(t)=\lambda\left(v_{k-1}(t)-v_{k}(t)\right)-\left(1-v_{k+1}(t)\right)^{d}+\left(1-v_{k}(t)\right)^{d} \quad k=1,2 \cdots
$$

Proof. The sequence of processes $\mathbf{u}(\cdot): n=1,2, \cdots$ is tight in the Skorokhod space $D_{U}[0, \infty)$ of right continuous functions with left limits in $U$ [2, Chapter 3.5]. Therefore every subsequence has a further subsequence that converges in distribution. By Skorokhod's Embedding Theorem [2, Theorem 3.1.8] the processes can be reconstructed in an appropriate probability space if necessary so that the convergence occurs almost surely. Since jumps of $\mathbf{u}(\cdot)$ have magnitudes that scale with $n^{-1}$, the limit process is continuous and convergence of $\mathbf{u}(\cdot)$ can be taken uoc [2, Theorem 3.10.1]. To describe a limit process $\mathbf{v}(\cdot)$ note that $u_{0}(t) \equiv 1$, and so $\varepsilon_{0}(t) \equiv 0$. For $k=1,2, \cdots$ the martingale $\varepsilon_{k}(\cdot)$ is square integrable. This process has $O(n)$ jumps per unit time and each jump is of size $n^{-1}$, hence its quadratic variation vanishes as $n \rightarrow \infty$. In turn, Doob's $L^{2}$ inequality [2, Proposition 2.2.16] implies that $\varepsilon_{k}(\cdot) \rightarrow 0$ uoc as $n \rightarrow \infty$. Since $\alpha_{n} \rightarrow 1$ and convergence of $\mathbf{u}(\cdot)$ is uoc, the $k$ th integral in equality (4) converges to

$$
\int_{0}^{t}\left(\lambda\left(v_{k-1}(s)-v_{k}(s)\right) d s-\left(\left(1-v_{k+1}(s)\right)^{d}-\left(1-v_{k}(s)\right)^{d}\right)\right) d s .
$$

Therefore $\mathbf{v}(\cdot)$ satisfies equality (4) with $\alpha_{n}=1$ and $\boldsymbol{\varepsilon}(t) \equiv \mathbf{0}$. Differential representation of that equality is (5).

Let $U_{o}$ denote the set of system states in which average queue occupancy is finite. That is,

$$
U_{o}=\left\{\mathbf{u} \in U: \sum_{k=1}^{\infty} u_{k}<\infty\right\} .
$$

Let $\mathbf{v}^{*}=\left\{v_{k}^{*}\right\}_{k=0}^{\infty} \in U$ be defined by setting $v_{0}^{*}=1$ and

$$
v_{k}^{*}=1-\sqrt[d]{1-\lambda v_{k-1}^{*}}, \quad k=1,2, \cdots
$$

Since $1-\sqrt[d]{1-\lambda v_{k-1}^{*}} \leq \lambda v_{k-1}^{*}$ it follows that $v_{k}^{*} \leq \lambda^{k}$; in particular $\mathbf{v}^{*} \in U_{o}$. It can be readily verified by substitution that $\mathbf{v}^{*}$ is an equilibrium point for the differential system (5). The following lemma establishes that $\mathbf{v}^{*}$ is the unique stable equilibrium for trajectories that start in $U_{o}$. 
Lemma 3.1 Let $\mathbf{v}(\cdot)$ solve the differential system (5) with initial state $\mathbf{v}(0) \in U_{o}$. Then

$$
\lim _{t \rightarrow \infty} v_{k}(t)=v_{k}^{*}, \quad k=1,2, \cdots
$$

We provide a proof based on the following auxiliary result:

Lemma 3.2 Let $\mathbf{v}^{+}(\cdot)$ and $\mathbf{v}^{-}(\cdot)$ solve the differential system (5) with respective initial conditions $\mathbf{v}^{+}(0), \mathbf{v}^{-}(0) \in U$ such that $v_{k}^{+}(0) \geq v_{k}^{-}(0)$ for all $k$. Then $v_{k}^{+}(t) \geq v_{k}^{-}(t)$ for all $k$ and all $t>0$.

Proof. Suppose that the lemma is incorrect and let $t>0$ be the first instant such that

$$
v_{k}^{+}(t)=v_{k}^{-}(t) \quad \text { and } \quad \frac{d}{d t} v_{k}^{+}(t)<\frac{d}{d t} v_{k}^{-}(t) \quad \text { for some } k \text {. }
$$

Let $i$ be the largest index $k$ that satisfies this condition at time $t$. Then by (5)

$$
\frac{d}{d t} v_{i}^{+}(t)-\frac{d}{d t} v_{i}^{-}(t)=\lambda\left(v_{i-1}^{+}(t)-v_{i-1}^{-}(t)\right)+\left(1-v_{i+1}^{-}(t)\right)^{d}-\left(1-v_{i+1}^{+}(t)\right)^{d} .
$$

The right hand side of this equality is nonnegative due to the choice of $t$ (since otherwise either the condition $v_{i-1}^{+}(t) \geq v_{i-1}^{-}(t)$ and or the condition $v_{i+1}^{+}(t) \geq v_{i+1}^{-}(t)$ must be violated before time $\left.t\right)$. This contradicts with the definition of $t$; therefore no such $t$ exists and the lemma holds.

Proof of Lemma 3.1 Let $\mathbf{v}^{+}(\cdot)$ and $\mathbf{v}^{-}(\cdot)$ be solutions to (5) with respective initial states $\mathbf{v}^{+}(0)$ and $\mathbf{v}^{-}(0)$ that are defined by setting $v_{k}^{+}(0)=\max \left\{v_{k}(0), v_{k}^{*}\right\}$ and $v_{k}^{-}(0)=\min \left\{v_{k}(0), v_{k}^{*}\right\}$ for $k=0,1,2, \cdots$. By Lemma 3.2

$$
v_{k}^{-}(t) \leq v_{k}(t), v_{k}^{*} \leq v_{k}^{+}(t), \quad \text { for all } k, t
$$

Equality (5) and definition (6) of $\mathbf{v}^{*}$ give

$$
\begin{aligned}
\frac{d}{d t} \sum_{i=k}^{\infty} v_{i}^{ \pm}(t) & =\lambda v_{k-1}^{ \pm}(t)+\left(1-v_{k}^{ \pm}(t)\right)^{d}-1 \\
& =\lambda\left(v_{k-1}^{ \pm}(t)-v_{k-1}^{*}\right)+\left(1-v_{k}^{ \pm}(t)\right)^{d}-\left(1-v_{k}^{*}\right)^{d}
\end{aligned}
$$

or, in integral form,

$$
\sum_{i=k}^{\infty} v_{i}^{ \pm}(t)-\sum_{i=k}^{\infty} v_{i}^{ \pm}(0)=\int_{0}^{t} \lambda\left(v_{k-1}^{ \pm}(s)-v_{k-1}^{*}\right) d s+\int_{0}^{t}\left(\left(1-v_{k}^{ \pm}(s)\right)^{d}-\left(1-v_{k}^{*}\right)^{d}\right) d s .
$$


Note that since $v_{1}^{+}(t) \geq v_{1}^{*}=1-\sqrt[d]{1-\lambda}$ it follows that

$$
\frac{d}{d t} \sum_{i=1}^{\infty} v_{k}^{+}(t)=\lambda+\left(1-v_{1}^{+}(t)\right)^{d}-1 \leq 0 .
$$

Hence $\sum_{i=k}^{\infty} v_{i}^{+}(t)$, and therefore $\sum_{i=k}^{\infty} v_{i}^{-}(t)$, is bounded by $\sum_{k=1}^{\infty} v_{k}^{+}(0)$ uniformly for all $t$. In turn equality (9) yields

$$
\left|\int_{0}^{t} \lambda\left(v_{k-1}^{ \pm}(s)-v_{k-1}^{*}\right) d s+\int_{0}^{t}\left(\left(1-v_{k}^{ \pm}(s)\right)^{d}-\left(1-v_{k}^{*}\right)^{d}\right) d s\right| \leq \sum_{i=k}^{\infty} v_{i}^{+}(0) .
$$

The bound on the right hand side is finite since $\mathbf{v}^{+}(0) \in U_{o}$ due to the hypothesis $\mathbf{v}(0) \in U_{o}$. Note that, owing to the inequality (8), neither one of the two integrands above changes sign. Hence if the first integral converges as $t \rightarrow \infty$ then so does the second one, implying further that

$$
\lim _{t \rightarrow \infty} v_{k}^{ \pm}(t)=v_{k}^{*}
$$

Since $v_{0}^{ \pm}(t) \equiv v_{0}^{*}=1$, this is clearly the case for $k=1$. Induction on $k$ confirms that equality (10) holds for all $k$. The desired conclusion (7) now follows from the property (8).

Theorem 3.1, which establishes convergence over finite time intervals, is complemented next by showing that equilibrium distribution of $\mathbf{u}(\cdot)$ converges as $n \rightarrow \infty$ to the deterministic measure concentrated at $\mathbf{v}^{*}$.

Theorem 3.2 The process $\mathbf{u}(\cdot)$ is ergodic. Let $\mathbf{u}^{*}=\left\{u_{k}^{*}\right\}_{k=0}^{\infty}$ denote the equilibrium random variable. For $\varepsilon>0$

$$
\lim _{n \rightarrow \infty} P_{n}\left(\rho\left(\mathbf{u}^{*}, \mathbf{v}^{*}\right)>\varepsilon\right)=0 .
$$

In particular $\lim _{n \rightarrow \infty} E_{n}\left[u_{k}^{*}\right]=v_{k}^{*}$ for $k=0,1,2, \cdots$.

Proof. Let $U_{n}=\left\{\mathbf{u} \in U: n u_{k} \in \mathbb{Z}_{+}\right.$for $\left.k \geq 0\right\}$. Note that $(\mathbf{u}(t): t \geq 0)$ is irreducible in $U_{n}$ and $U_{n}$ is compact; therefore the process is ergodic and has a unique equilibrium distribution concentrated on $U_{n}$. In that equilibrium the rate of arrivals to queues with occupancy $k$ or higher should be equal to the rate of departures from such queues. That is,

$$
E_{n}\left[\lambda u_{k-1}^{*}\right]=1-E_{n}\left[\left(1-u_{k}^{*}\right)^{d}\right] \geq E_{n}\left[u_{k}^{*}\right], \quad \text { for } k \geq 1
$$

where the inequality follows since $\left(1-u_{k}^{*}\right)^{d} \leq\left(1-u_{k}^{*}\right)$. Therefore $E_{n}\left[u_{k}^{*}\right] \leq \lambda^{k}$ and in turn $E_{n}\left[\sum_{k=1}^{\infty} u_{k}^{*}\right] \leq \lambda /(1-\lambda)$. Let $U_{o, \lambda} \triangleq\left\{\mathbf{u} \in U: \sum_{k=1}^{\infty} u_{k} \leq \lambda /(1-\lambda)\right\}$ so that $P_{n}\left(\mathbf{u}^{*} \in U_{o, \lambda}\right)=1$. 
Suppose that (11) is false so that for some infinite subsequence $\left\{n^{\prime}\right\}$ of $\{n\}$ and some $\delta>0$

$$
P_{n^{\prime}}\left(\rho\left(\mathbf{u}^{*}, \mathbf{v}^{*}\right)>\varepsilon\right)>\delta
$$

Due to Lemma 3.1 and compactness of $U_{o, \lambda}$ one can choose $t(\varepsilon)$ such that if $\mathbf{v}(0) \in U_{o, \lambda}$ then $\rho\left(\mathbf{v}(t), \mathbf{v}^{*}\right)<\varepsilon / 2$ for $t \geq t(\varepsilon)$. Let $\mathbf{u}(0)$ have the same distribution as $\mathbf{u}^{*}$ and let $\mathbf{v}(0)=\mathbf{u}(0)$. By Theorem 3.1 there is a further subsequence $\left\{n^{\prime \prime}\right\}$ of $\left\{n^{\prime}\right\}$ such that $P_{n^{\prime \prime}}(\rho(\mathbf{u}(t(\varepsilon)), \mathbf{v}(t(\varepsilon)))>$ $\varepsilon / 2)<\delta$ whenever $n^{\prime \prime}$ is large enough. Since $P_{n^{\prime \prime}}\left(\mathbf{u}^{*} \in U_{o, \lambda}\right)=1$, the choice of $t(\varepsilon)$ implies that $P_{n^{\prime \prime}}\left(\rho\left(\mathbf{u}(t(\varepsilon)), \mathbf{v}^{*}\right)>\varepsilon\right)<\delta$ for those values of $n^{\prime \prime}$. However $\mathbf{u}(t(\varepsilon))$ and $\mathbf{u}^{*}$ have identical distributions as the latter is in equilibrium; leading to a contradiction with (12). Hence no sequence $\left\{n^{\prime}\right\}$ and constant $\delta>0$ satisfy (12); so (11) holds. By definition of $\rho$ equality (11) implies that each entry $u_{k}^{*}$ of $\mathbf{u}^{*}$ converges in probability to the constant $v_{k}^{*}$; since $0 \leq u_{k}^{*} \leq 1$, so does $E_{n}\left[u_{k}^{*}\right]$.

We conclude the discussion of $\operatorname{LCQ}(d)$ with a relationship between $d$ and the tail probabilities of equilibrium queue occupancy:

Theorem $3.3 v_{k}^{*}=\Theta\left((\lambda / d)^{k}\right)$ as $k \rightarrow \infty$.

Proof. The assertion is immediate for $d=1$ so we consider the case $d>1$. Equality ([6]), together with Taylor expansion of $\sqrt[d]{1-x}$ around $x=0$ yields

$$
v_{k}^{*}=\frac{\lambda}{d} v_{k-1}^{*}+\frac{1}{d} \sum_{i=2}^{\infty} \frac{\left(\lambda v_{k-1}^{*}\right)^{i}}{i !} \prod_{j=1}^{i-1}\left(j-\frac{1}{d}\right), \quad k=1,2, \cdots
$$

The second term on the right hand side is nonnegative; therefore $v_{k}^{*} \geq(\lambda / d) v_{k}^{*}$ and

$$
\liminf _{k \rightarrow \infty} \frac{v_{k}^{*}}{(\lambda / d)^{k}} \geq 1
$$

We define $c_{k} \triangleq v_{k}^{*} /(\lambda / d)^{k}$ and complete the proof by showing that $\left\{c_{k}\right\}_{k=0}^{\infty}$ is uniformly bounded. Let $\beta_{k}$ be defined as

$$
\beta_{k} \triangleq 1+\sum_{i=1}^{\infty}\left(\lambda v_{k-1}^{*}\right)^{i} \frac{2}{(i+2) !} \prod_{j=2}^{i+1}\left(j-\frac{1}{d}\right)<1+\sum_{i=1}^{\infty}\left(\lambda v_{k-1}^{*}\right)^{i}
$$

so that equality (13) can be rearranged as

$$
v_{k}^{*}=\frac{\lambda}{d} v_{k-1}^{*}+\frac{\left(\lambda v_{k-1}^{*}\right)^{2}}{2 d}\left(1-\frac{1}{d}\right) \beta_{k} .
$$


Since $v_{k}^{*} \leq \lambda^{k}$ there exists a finite $k_{o}$ such that $v_{k-1}^{*}<1 / d$ for $k \geq k_{o}$. The bound in (15) implies that $\beta_{k}<1 /(1-\lambda / d)<d /(d-1)$ for such $k$; in turn by (16)

$$
c_{k}<c_{k-1}+c_{k-1}^{2} \frac{d}{2}(\lambda / d)^{k}, \quad k>k_{o} .
$$

It can be verified by induction on $k>k_{o}$ that

$$
c_{k}<c_{k_{o}}\left(1+\lambda+\lambda^{2}+\cdots+\lambda^{k-k_{o}}\right):
$$

Namely, if (17) holds for $k$ then it holds also for $k+1$ if $c_{k_{o}}\left(1+\lambda+\lambda^{2}+\cdots+\lambda^{k-k_{o}}\right)^{2} \lambda^{k_{o}} /\left(2 d^{k}\right)<1$. This latter condition can be verified based on the bound $c_{k_{o}}(\lambda / d)^{k_{o}}=v_{k_{o}}<1 / d$, which follows from the definition of $k_{o}$. Inequality (17) implies the uniform bound $c_{k}<c_{k_{o}} /(1-\lambda)$; therefore

$$
\limsup _{k \rightarrow \infty} \frac{v_{k}^{*}}{(\lambda / d)^{k}}<\frac{c_{k_{o}}}{1-\lambda}
$$

The theorem follows due to (14) and (18).

\section{LCQ}

Given $\mathbf{m}(\tau)$ at a scheduling instant $\tau$, the maximum occupancy over all connected queues at time $\tau$ is equal to $k=1,2, \cdots$ with probability $(1-q)^{m_{k+1}(\tau)}-(1-q)^{m_{k}(\tau)}$; hence under the LCQ policy $\mathbf{u}(\cdot)$ is a time-homogenous Markov process with jump rates

$$
\mathbf{u} \leftarrow\left\{\begin{array}{lll}
\mathbf{u}+n^{-1} \mathbf{e}_{k} & \text { at rate } & n \lambda\left(u_{k-1}-u_{k}\right) \\
\mathbf{u}-n^{-1} \mathbf{e}_{k} & \text { at rate } & n\left((1-q)^{n u_{k+1}}-(1-q)^{n u_{k}}\right) .
\end{array}\right.
$$

This process can be constructed as in Section 3, so that

$$
u_{k}(t)=u_{k}(0)+\int_{0}^{t}\left(\lambda\left(u_{k-1}(s)-u_{k}(s)\right)-(1-q)^{m_{k+1}(s)}+(1-q)^{m_{k}(s)}\right) d s+\varepsilon_{k}(t)
$$

where $\varepsilon_{k}(\cdot)$ is a martingale that vanishes as $n \rightarrow \infty$. The sequence of processes $\mathbf{u}(\cdot): n=1,2, \cdots$ converges in distribution along subsequences of $\{n\}$, but identifying a limit is relatively more involved than for the LCQ $(d)$ policy since the process $\mathbf{m}(\cdot)$ fluctuates persistently for all values of $n$ and the integrand in (20) does not converge. Rather than this integrand, here we study the behavior of the integral in (201) via an averaging technique due to Kurtz [5]. In reading this section the reader may

find it helpful to consult related applications of this technique in [1, 4, 13. 
Let $\Omega$ denote the set of sequences $\boldsymbol{\omega}=\left\{\omega_{k}\right\}_{k=0}^{\infty}$ such that $\omega_{k} \in \mathbb{Z}_{+} \cup\{\infty\}, \omega_{0}=\infty$, and $\omega_{k} \geq \omega_{k+1}$. Define the mapping $h: \Omega \mapsto[0,1]^{\infty}$ by setting

$$
h(\boldsymbol{\omega})=\left\{\left(1+\omega_{k}\right)^{-1}\right\}_{k=0}^{\infty}, \quad \boldsymbol{\omega} \in \Omega
$$

with the understanding that $1+\infty=\infty$ and $1 / \infty=0$. Let $\Omega$ be endowed with metric $\rho_{o}$ defined by

$$
\rho_{o}\left(\boldsymbol{\omega}, \boldsymbol{\omega}^{\prime}\right)=\rho\left(h(\boldsymbol{\omega}), h\left(\boldsymbol{\omega}^{\prime}\right)\right), \quad \boldsymbol{\omega}, \boldsymbol{\omega}^{\prime} \in \Omega
$$

In particular $\Omega$ is compact with respect to the induced topology. We denote by $\mathcal{L}$ the collection of measures $\mu$ on the product space $[0, \infty) \times \Omega$ such that $\mu([0, t) \times \Omega)=t$ for each $t>0$. Let $\mathcal{L}$ be endowed with the topology corresponding to weak convergence of measures restricted to $[0, t) \times \Omega$ for each $t$. Since $\Omega$ is compact, so is $\mathcal{L}$ due to Prohorov's Theorem.

Let $\xi$ be a random member of $\mathcal{L}$ defined by

$$
\xi([0, t) \times A)=\int_{0}^{t} 1\{\mathbf{m}(s) \in A\} d s, \quad t>0, A \in \mathcal{B}(\Omega) .
$$

Here $\mathcal{B}(\Omega)$ denotes Borel sets of $\Omega$. Note that equality (20) can be expressed in terms of $\xi$ as

$$
u_{k}(t)=u_{k}(0)+\int_{0}^{t} \lambda\left(u_{k-1}(s)-u_{k}(s)\right) d s-\phi_{k+1}(t)+\phi_{k}(t)+\varepsilon_{k}(t)
$$

where

$$
\phi_{k}(t) \triangleq \int_{0}^{t}(1-q)^{m_{k}(s)} d s=\int_{[0, t) \times \Omega}(1-q)^{\omega_{k}} \xi(d s \times d \boldsymbol{\omega}) .
$$

Compactness of $\mathcal{L}$ implies that each subsequence of $\{n\}$ has a further subsequence along which $\xi$ converges in distribution. This property is also possessed by $\mathbf{u}(\cdot)$, and therefore by the pair $(\mathbf{u}(\cdot), \xi)$.

The following definition is useful in characterizing possible limits of $(\mathbf{u}(\cdot), \xi)$ : For fixed $\mathbf{u} \in U$ let $\omega^{\mathbf{u}}(\cdot)$ denote the Markov process with states in $\Omega$ and with the following transition rates:

$$
\omega^{\mathbf{u}} \leftarrow \begin{cases}\omega^{\mathbf{u}}+\mathbf{e}_{k} & \text { at rate } \lambda\left(u_{k-1}-u_{k}\right) \\ \omega^{\mathbf{u}}-\mathbf{e}_{k} & \text { at rate }(1-q)^{\omega_{k+1}^{\mathbf{u}}}-(1-q)^{\omega_{k}^{\mathbf{u}}}\end{cases}
$$

See Figure 3 for a partial illustration of this process. The process $\omega^{\mathbf{u}}(\cdot)$ bears a certain resemblance to $\mathbf{m}(\cdot)=n \mathbf{u}(\cdot)$, which can be observed by inspecting the generators (19) and (21), though it should be noted that in (21) $\mathbf{u}=\left\{u_{k}\right\}_{k=0}^{\infty}$ is a constant and has no binding to instantaneous values of $\omega^{\mathbf{u}}(\cdot)$. We also point out that $\omega^{\mathbf{u}}(\cdot)$ evolves on a compactified state space and it is reducible due to the states that involve $\infty$; hence it has multiple equilibrium distributions in general. 


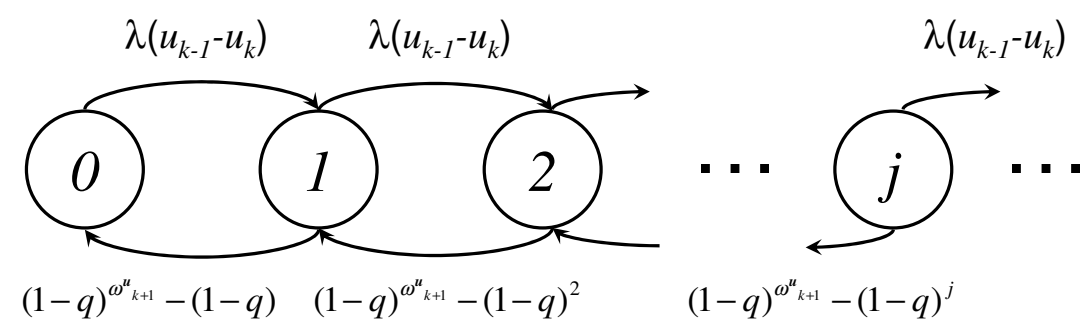

Figure 3: Transition rates of $\omega_{k}^{\mathbf{u}}(\cdot)$, that is, the $k$ th coordinate of $\omega^{\mathbf{u}}(\cdot)$. The process has also an isolated state $\infty$ which is not shown. The coordinate process $\omega_{k}^{\mathbf{u}}(\cdot)$ is generally not Markovian due to its dependence on $\omega_{k+1}^{\mathbf{u}}(\cdot)$.

Theorem 4.1 Let $(\mathbf{v}(\cdot), \chi)$ be the limit of $(\mathbf{u}(\cdot), \xi)$ along a convergent subsequence of $\{n\}$.

a) The limit measure $\chi$ satisfies

$$
\chi([0, t) \times A)=\int_{0}^{t} \pi_{\mathbf{v}(s)}(A) d s, \quad t>0, A \in \mathcal{B}(\Omega),
$$

where, for each $s>0, \pi_{\mathbf{v}(s)}$ is an equilibrium distribution for the process $\omega^{\mathbf{v}(s)}(\cdot)$ such that

$$
\pi_{\mathbf{v}(s)}\left(\omega_{k}=\infty\right)=1 \quad \text { if } \quad v_{k}(s)>0
$$

b) The limit trajectory $\mathbf{v}(\cdot)$ satisfies

$$
\frac{d}{d t} v_{k}(t)=\lambda\left(v_{k-1}(t)-v_{k}(t)\right)-E_{\pi_{\mathbf{v}(t)}}\left[(1-q)^{\omega_{k+1}}-(1-q)^{\omega_{k}}\right]
$$

where $k=1,2, \cdots$ and $E_{\pi_{\mathbf{v}(t)}}$ denotes expectation with respect to distribution $\pi_{\mathbf{v}(t)}$.

Proof. Let all processes be constructed on a common probability space so that convergence of $(\mathbf{u}(\cdot), \xi)$ is almost sure. Convergence of $\mathbf{u}(\cdot)$ is then uoc. We start by consulting [5, Lemma 1.4] to verify that the limit measure $\chi$ possesses a density so that

$$
\chi([0, t) \times A)=\int_{0}^{t} \gamma_{s}(A) d s, \quad t>0, A \in \mathcal{B}(\Omega),
$$

where, for each $s, \gamma_{s}$ is a probability distribution on $\Omega$. We proceed by identifying these distributions.

Let $\mathcal{F}$ denote the collection of bounded continuous functions $f: \Omega \mapsto \mathbb{R}$ such that $f(\boldsymbol{\omega})$ depends on a finite number of entries in the sequence $\boldsymbol{\omega}=\left\{\omega_{k}\right\}_{k=0}^{\infty} \in \Omega$. Given $f \in \mathcal{F}$ define the function 
$G^{f}: \Omega \times U \mapsto \mathbb{R}$ by setting

$$
G^{f}(\boldsymbol{\omega}, \mathbf{u}) \triangleq \sum_{k=1}^{\infty}\left(f\left(\boldsymbol{\omega}+\mathbf{e}_{k}\right)-f(\boldsymbol{\omega})\right) \lambda\left(u_{k-1}-u_{k}\right)+\left(f\left(\boldsymbol{\omega}-\mathbf{e}_{k}\right)-f(\boldsymbol{\omega})\right)\left((1-q)^{\omega_{k+1}}-(1-q)^{\omega_{k}}\right)
$$

for each $\boldsymbol{\omega} \in \Omega$ and $\mathbf{u}=\left\{u_{k}\right\}_{k=0}^{\infty} \in U$. $G^{f}$ is continuous due to the continuity of $f$, and continuity of $G^{f}$ is uniform since the product space $\Omega \times U$ is compact.

The process $f(\mathbf{m}(\cdot))$ satisfies at each instant $t$

$$
\begin{aligned}
f(\mathbf{m}(t))-f(\mathbf{m}(0)) & \int_{0}^{t} \sum_{k=1}^{\infty}\left(f\left(\mathbf{m}(s)+\mathbf{e}_{k}\right)-f(\mathbf{m}(s))\right) d A_{k-1}\left(\int_{0}^{s} n \lambda\left(u_{k-1}(\tau)-u_{k}(\tau)\right) d \tau\right) \\
& +\int_{0}^{t} \sum_{k=1}^{\infty}\left(f\left(\mathbf{m}(s)-\mathbf{e}_{k}\right)-f(\mathbf{m}(s))\right) d D_{k}\left(\int_{0}^{s} n\left((1-q)^{m_{k+1}(\tau)}-(1-q)^{m_{k}(\tau)}\right) d \tau\right) \\
= & n \int_{0}^{t} \sum_{k=1}^{\infty}\left(f\left(\mathbf{m}(s)+\mathbf{e}_{k}\right)-f(\mathbf{m}(s))\right) \lambda\left(u_{k-1}(s)-u_{k}(s)\right) d s \\
& +n \int_{0}^{t} \sum_{k=1}^{\infty}\left(f\left(\mathbf{m}(s)-\mathbf{e}_{k}\right)-f(\mathbf{m}(s))\right)\left((1-q)^{m_{k+1}(s)}-(1-q)^{m_{k}(s)}\right) d s+\mu^{f}(t) \\
= & n \int_{0}^{t} G^{f}(\mathbf{m}(s), \mathbf{u}(s)) d s+\mu^{f}(t),
\end{aligned}
$$

where $\mu^{f}(\cdot)$ is a square-integrable martingale. Rearranging the last equality and expressing the integral there in terms of the random measure $\xi$ yields

$$
\int_{[0, t) \times \Omega} G^{f}(\boldsymbol{\omega}, \mathbf{u}(s)) \xi(d s \times d \boldsymbol{\omega})=\int_{0}^{t} G^{f}(\mathbf{m}(s), \mathbf{u}(s)) d s=\left(f(\mathbf{m}(t))-f(\mathbf{m}(0)) / n+\mu^{f}(t) / n .\right.
$$

Since $f$ is bounded, the first term on the right hand side vanishes as $n \rightarrow \infty$. The martingale $\mu^{f}(\cdot)$ has bounded jumps; in turn by Doob's $L^{2}$ inequality $\mu^{f}(t) / n$ also vanishes. Therefore

$$
\int_{[0, t) \times \Omega} G^{f}(\boldsymbol{\omega}, \mathbf{u}(s)) \xi(d s \times d \boldsymbol{\omega}) \rightarrow 0 .
$$

Since $\mathbf{u}(\cdot)$ converges uoc to $\mathbf{v}(\cdot)$ by hypothesis, uniform continuity of $G^{f}$ implies

$$
\left|\int_{[0, t) \times \Omega} G^{f}(\boldsymbol{\omega}, \mathbf{u}(s)) \xi(d s \times d \boldsymbol{\omega})-\int_{[0, t) \times \Omega} G^{f}(\boldsymbol{\omega}, \mathbf{v}(s)) \xi(d s \times d \boldsymbol{\omega})\right| \rightarrow 0 .
$$

Finally by the Continuous Mapping Theorem

$$
\int_{[0, t) \times \Omega} G^{f}(\boldsymbol{\omega}, \mathbf{v}(s)) \xi(d s \times d \boldsymbol{\omega}) \rightarrow \int_{[0, t) \times \Omega} G^{f}(\boldsymbol{\omega}, \mathbf{v}(s)) \chi(d s \times d \boldsymbol{\omega}) .
$$

Observations (24)-(26) lead to

$$
\int_{[0, t) \times \Omega} G^{f}(\boldsymbol{\omega}, \mathbf{v}(s)) \chi(d s \times d \boldsymbol{\omega})=\int_{0}^{t} \sum_{\boldsymbol{\omega} \in \Omega} G^{f}(\boldsymbol{\omega}, \mathbf{v}(s)) \gamma_{s}(\boldsymbol{\omega}) d s=0
$$


where the left equality is due to (23). This equality holds for all $t>0$; therefore

$$
\sum_{\boldsymbol{\omega} \in \Omega} G^{f}(\boldsymbol{\omega}, \mathbf{v}(s)) \gamma_{s}(\boldsymbol{\omega})=0
$$

for almost all $s>0$. Since $f \in \mathcal{F}$ is arbitrary (note that $\mathcal{F}$ is dense in continuous bounded functions on $\Omega$ ) [2, Proposition 4.9.2] implies that $\gamma_{s}$ is an equilibrium distribution for the process $\boldsymbol{\omega}^{\mathbf{v}(s)}(\cdot)$.

Let $\varepsilon>0$ and $\left[t_{0}, t_{1}\right]$ be an interval such that $v_{k}(t) \geq \varepsilon$ for $t \in\left[t_{0}, t_{1}\right]$. Since $v_{k}(t)$ is the limit of $u_{k}(t)=n^{-1} m_{k}(t)$, for any given integer $B$

$$
\xi\left(\left[t_{0}, t_{1}\right] \times\{0,1,2, \cdots, B\}\right)=\int_{t_{0}}^{t_{1}} 1\left\{m_{k}(s) \leq B\right\} d s \rightarrow 0 \quad \text { as } n \rightarrow \infty .
$$

Hence $\chi\left(\left[t_{0}, t_{1}\right] \times \mathbb{Z}_{+}\right)=0$ due to the arbitrariness of $B$. Since $\varepsilon$ can be chosen arbitrarily small it follows that $\gamma_{t}\left(\mathbb{Z}_{+}\right)=0$ for almost all $t$ such that $v_{k}(t)>0$. This completes the proof of part a). Part b) follows from equality (20) since

$$
\int_{0}^{t}\left(u_{k-1}(s)-u_{k}(s)\right) d s \rightarrow \int_{0}^{t}\left(v_{k-1}(s)-v_{k}(s)\right) d s
$$

due to uoc convergence of $\mathbf{u}(\cdot)$, and

$$
\phi_{k}(t) \rightarrow \int_{[0, t) \times \Omega}(1-q)^{\omega_{k}} \chi(d s \times d \boldsymbol{\omega})=\int_{0}^{t} E_{\gamma_{s}}\left[(1-q)^{w_{k}}\right] d s
$$

due to the Continuous Mapping Theorem.

Theorem 4.1 explains the extent of the disparity between time scales of two processes, namely $\mathbf{m}(\cdot)$ and its normalized version $\mathbf{u}(\cdot)$ : The process $\mathbf{m}(\cdot)$ displays far larger variation than its normalized version, so that, in the limit of large $n, \mathbf{m}(\cdot)$ settles to equilibrium before $\mathbf{u}(\cdot)$ changes its value. In particular integral of a binary-valued measurable function of $\mathbf{m}(\cdot)$ is well-approximated by integrating an appropriate equilibrium probability. Provided that $\mathbf{u}(t)$ remains close to $\mathbf{v}(t)$, the process $\boldsymbol{\omega}^{\mathbf{v}(t)}(\cdot)$ mimics a slowed-down version of $\mathbf{m}(\cdot)$ observed around time $t$; hence the alluded equilibrium distribution pertains to $\omega^{\mathbf{v}(t)}(\cdot)$.

Specification of $\boldsymbol{\omega}^{\mathbf{v}(t)}(\cdot)$ requires inclusion of $\infty$ since entries of $\mathbf{m}(\cdot)$ can be as large as $n$. Compactifying the augmented state-space $\Omega$ of $\mathbf{m}(\cdot)$ via choice of the metric $\rho_{o}$ leads to the representation (22) of a limit trajectory $\mathbf{v}(\cdot)$, but it also entails ambiguity in that representation. Namely, Theorem 4.1 does not specify which equilibrium distribution for $\omega^{\mathbf{v}(t)}(\cdot)$ should be adopted in (22). While a full account of equilibrium distributions of $\omega^{\mathbf{v}(t)}(\cdot)$ appears difficult, an important feature of the right distribution can be identified: 
Lemma 4.1 Let $\mathbf{v}(\cdot)$ and $\pi_{\mathbf{v}(\cdot)}$ be as specified by Theorem 4.1. Given $k=1,2, \cdots$

$$
\pi_{\mathbf{v}(t)}\left(\omega_{k} \in \mathbb{Z}_{+} \text {and } \omega_{k+1}=0\right)=1
$$

for almost all $t$ such that $v_{k}(t)=0$.

Lemma 4.1 will be instrumental in obtaining a sharper description for $\mathbf{v}(\cdot)$, yet an informal explanation may still be useful in putting it in perspective with the queueing system of interest. Note that if $v_{k}(t)=0$ and $v_{k-1}(t)>0$ then $\mathbf{v}(t)$ reflects a distribution with support $\{0,1, \cdots, k-1\}$. This property does not immediately translate into a bound on the maximum queue length in the system, since $\mathbf{v}(t)$ is the limit of $\mathbf{u}(t)=n^{-1} \mathbf{m}(t)$ and so the number of queues with at least $i \geq k$ packets, $m_{i}(t)$, is $o(n)$ as $n \rightarrow \infty$. By way of interpreting $\boldsymbol{\omega}^{\mathbf{v}(t)}(\cdot)$ as a proxy to $\mathbf{m}(\cdot)$ around time $t$, Lemma 4.1 indicates that the maximum queue size is at most one larger than what is deduced from $\mathbf{v}(t)$ and that the number of maximal queues is $O(1)$ as $n \rightarrow \infty$.

Proof of Lemma 4.1 Let $\left[t_{0}, t_{1}\right]$ be an interval such that $v_{k}(t)=0$ for $t \in\left[t_{0}, t_{1}\right]$. We prove the lemma by showing that as $n \rightarrow \infty$ along the convergent subsequence of interest

$$
\begin{aligned}
\xi\left(\left[t_{0}, t_{1}\right] \times\left\{\boldsymbol{\omega}: w_{k+1}=0\right\}\right) & =\int_{t_{0}}^{t_{1}} 1\left\{m_{k+1}(t) \geq 1\right\} d t \rightarrow 0 \\
\xi\left(\left[t_{0}, t_{1}\right] \times\left\{\boldsymbol{\omega}: w_{k} \in Z_{+}\right\}\right) & =\int_{t_{0}}^{t_{1}} 1\left\{m_{k}(t) \in Z_{+}\right\} d t \rightarrow t_{1}-t_{0} .
\end{aligned}
$$

For each integer $l$ and time $t$ let $s_{l}(t) \triangleq \sum_{i=l}^{\infty} m_{i}(t)$. This quantity increases when some queue with size at least $l-1$ receives a packet, and it decreases when transmission is scheduled from some queue with size at least $l$. Given $\mathbf{u}(t)$, these events occur at respective instantaneous rates $n \lambda u_{l-1}(t)$ and $n\left(1-(1-q)^{m_{l}(t)}\right)$. Therefore

$$
E_{n}\left[s_{l}\left(t_{1}\right)-s_{l}\left(t_{0}\right)\right]=n E_{n}\left[\int_{t_{0}}^{t_{1}} \lambda u_{l-1}(t)-\left(1-(1-q)^{m_{l}(t)}\right) d t\right] .
$$

Consider this equality for $l=k+1$. By choice of the interval $\left[t_{0}, t_{1}\right]$

$$
n^{-1} E_{n}\left[s_{k+1}(t)\right] \rightarrow \sum_{i=k+1}^{\infty} v_{i}(t)=0
$$

and $u_{k}(t) \rightarrow v_{k}(t)=0$ for all $t \in\left[t_{0}, t_{1}\right]$. Consequently

$$
E_{n}\left[\int_{t_{0}}^{t_{1}}\left(1-(1-q)^{m_{k+1}(t)}\right) d t\right] \rightarrow 0
$$


This leads to (27) since

$$
1-(1-q)^{m_{k+1}(t)} \geq q 1\left\{m_{k+1}(t) \geq 1\right\}
$$

To complete the proof, note that $n^{-1} E_{n}\left[s_{k}(t)\right] \rightarrow 0$ for all $t \in\left[t_{0}, t_{1}\right]$; therefore (29) evaluated at $l=k$ implies that for any open subset $B \subset\left[t_{0}, t_{1}\right]$

$$
\limsup _{n \rightarrow \infty} E_{n}\left[\int_{B}\left(1-(1-q)^{m_{k}(t)}\right) d t\right]=\limsup _{n \rightarrow \infty} E_{n}\left[\int_{B} \lambda u_{k-1}(t) d t\right]<\int_{B} d t
$$

The last inequality is strict since $\lambda<1$. Arbitrariness of $B$ implies (28).

Given positive integer $K$ let $U_{K}=\left\{\mathbf{u} \in U: u_{k}=0\right.$ for $\left.k \geq K\right\}$.

Theorem 4.2 Let $\mathbf{v}(\cdot)$ and $\pi_{\mathbf{v}(\cdot)}$ be as specified by Theorem 4.1 with initial state $\mathbf{v}(0) \in U_{K}$ for some $K$. Then for $t>0$

a) $\mathbf{v}(t) \in U_{K}$ and

$$
\pi_{\mathbf{v}(t)}\left(\omega_{K(t)} \in \mathbb{Z}_{+} \text {and } \omega_{K(t)+1}=0\right)=1
$$

where $K(t)=\min \left\{k: v_{j}(t)=0\right.$ for $\left.j \geq k\right\}$.

b)

$$
\frac{d}{d t} v_{k}(t)= \begin{cases}\lambda v_{k-1}(t)-1<0 & \text { if } k=K(t)-1 \\ 0 & \text { if } k \geq K(t) .\end{cases}
$$

In particular $v_{k}(t)=0$ for $k>0$ and $t>K /(1-\lambda)$.

Proof. Let $t$ be an instant such that $K(t)<\infty$. Lemma 4.1 implies that

$$
\pi_{\mathbf{v}(t)}\left(\omega_{K(t)} \in \mathbb{Z}_{+} \text {and } \omega_{K(t)+1}=0\right)=1
$$

In particular the coordinate process $\boldsymbol{\omega}_{K(t)}^{\mathbf{v}(t)}(\cdot)$ possesses an equilibrium in $\mathbb{Z}_{+}$. The process should have equal rates of up-jumps and down-jumps in that equilibrium, namely

$$
E_{\pi_{\mathbf{v}(t)}}\left[(1-q)^{\omega_{K(t)}}\right]=1-\lambda v_{K(t)-1}(t)
$$

Since $v_{K(t)-1}(t)>0$ by definition of $K(t)$, Theorem 4.1,a implies that

$$
E_{\pi_{\mathbf{v}(t)}}\left[(1-q)^{\omega_{K(t)-1}}\right]=0 .
$$


Substituting (31) and (32) in equality (22) evaluated at $k=K(t)-1$ yields

$$
\frac{d}{d t} v_{K(t)-1}(t)=\lambda v_{K(t)-2}(t)-1<0
$$

Note also that $E_{\pi_{\mathbf{v}(t)}}\left[(1-q)^{\omega_{K(t)+1}}\right]=1$ due to (30); hence equality (22) for $k=K(t)$ gives

$$
\frac{d}{d t} v_{K(t)}(t)=-\lambda v_{K(t)}(t)=0
$$

Since $K(0)=K<\infty$ by hypothesis, it follows via (33) and (34) that $K(t)$ is finite and nonincreasing in $t$. Part (a) of the theorem now follows by (30). Part (b) is due to (33) and (34).

Corollary 4.1 If $\mathbf{u}(0) \in U_{K}$ for some $K$ then

$$
\lim _{n \rightarrow \infty} P_{n}\left(m_{1}(t) \in \mathbb{Z}_{+}, m_{2}(t)=0\right)=1
$$

for $t \geq K /(1-\lambda)$. The system occupancy $\sum_{k=1}^{\infty} m_{k}(t)$ converges in distribution to the equilibrium value of a birth-death process with constant birth rate $\lambda$ and death rate $1-(1-q)^{j}$ at state $j$.

Proof. Let $\left\{n_{i}\right\}$ be a subsequence along which $(\mathbf{u}(\cdot), \xi)$ converges and let $(\mathbf{v}(\cdot), \chi)$ denote the limit. Since $\mathbf{u}(0) \in U_{K}$ it follows that $\mathbf{v}(0) \in U_{K}$. Choose $t_{1}>t_{0}>K /(1-\lambda)$ so that by Theorem 4.2,b $\mathbf{v}(t)=\{1,0,0,0, \cdots\}$ for $t \in\left[t_{0}, t_{1}\right]$. Let $A=\left\{\boldsymbol{\omega} \in \Omega: \omega_{1} \in \mathbb{Z}_{+}, w_{2}=0\right\}$. Then

$$
\int_{t_{0}}^{t_{1}} P_{n_{i}}(\mathbf{m}(t) \in A) d t=E_{n_{i}}\left[\int_{t_{0}}^{t_{1}} 1\{\mathbf{m}(t) \in A\} d t\right] \rightarrow \int_{t_{0}}^{t_{1}} \pi_{\mathbf{v}(t)}(A) d t=t_{1}-t_{0},
$$

where the last equality is due to Theorem 4.2, a. The above limit does not depend on the particular subsequence $\left\{n_{i}\right\}$; therefore (35) follows. The final claim of the corollary is verified by observing that for $t>K /(1-\lambda)$ the coordinate process $\omega_{2}^{\mathbf{v}(t)} \equiv 0$ in equilibrium; and in turn $\omega_{1}^{\mathbf{v}(t)}$ is a positive recurrent birth-death process on $\mathbb{Z}_{+}$with birth rate $\lambda$ and death rate $1-(1-q)^{j}$ at state $j$.

It should be noted that the hypothesis $\mathbf{u}(0) \in U_{K}$ is necessary for the conclusions of Corollary 4.1] If the initial size of a single queue is allowed to grow without bound with increasing $n$ then, for large values of $n$, that queue receives service whenever it is connected. In effect this reduces the service rate available to the rest of the system by a factor of $(1-q)$. In such degenerate cases the present analysis applies to the subsystem that is composed of queues with bounded initial occupancies, after appropriate adjustment of the service rate. 


\section{Final remarks: $\operatorname{LCQ}\left(d_{n}\right)$}

Conclusions of Sections 3 and 4 reveal that the system occupancies under LCQ $(d)$ and LCQ differ by a factor of order $n$ as $n \rightarrow \infty$. More insight on this disparity, especially for moderate values of $d$ relative to $n$, can be gained by considering an asymptotic regime in which $d$ is allowed to depend on $n$. Here we sketch asymptotic analysis of $\operatorname{LCQ}\left(d_{n}\right)$ in the case

$$
\lim _{n \rightarrow \infty} d_{n}=\infty \quad \text { and } \quad \lim _{n \rightarrow \infty} \frac{d_{n}}{n}=0
$$

The present discussion closely follows that of Section 4, hence proofs are omitted.

Under LCQ $\left(d_{n}\right)$ the representation (4) can be expressed as

$$
u_{k}(t)=u_{k}(0)+\int_{0}^{t}\left(\lambda\left(u_{k-1}(s)-u_{k}(s)\right)-\left(\left(1-\frac{b_{k+1}(s)}{d_{n}}\right)^{d_{n}}-\left(1-\frac{b_{k}(s)}{d_{n}}\right)^{d_{n}}\right)\right) d s+\varepsilon_{k}(t)
$$

where $b_{k}(t) \triangleq d_{n} u_{k}(t)$. Let $\mathbf{b}(t)=\left\{b_{k}(t)\right\}_{k=0}^{\infty}$ and let $\Omega_{o}$ be obtained by augmenting $\Omega$ with nonincreasing sequences that take values in $\mathbb{R}_{+} \cup\{\infty\}$. Define the random measure $\xi_{o}$ by

$$
\xi_{o}([0, t) \times A)=\int_{0}^{t} 1\{\mathbf{b}(s) \in A\} d s, \quad t>0, A \in \mathcal{B}\left(\Omega_{o}\right) .
$$

Consideration of the pair $\left(\mathbf{u}(\cdot), \xi_{o}\right)$ via an analogue of Theorem 4.1 identifies possible limits $\mathbf{v}(\cdot)$ of $\mathbf{u}(\cdot)$ as solutions to

$$
\frac{d}{d t} v_{k}(t)=\lambda\left(v_{k-1}(t)-v_{k}(t)\right)-E_{\pi_{\mathbf{v}(t)}}\left[e^{-\omega_{k+1}}-e^{-\omega_{k}}\right], \quad k=1,2, \cdots
$$

where $\pi_{\mathbf{v}(t)}$ is a distribution on $\Omega_{o}$ such that $\pi_{\mathbf{v}(t)}\left(\omega_{k}=\infty\right)=1$ if $v_{k}(t)>0$ and

$$
E_{\pi_{\mathbf{v}(t)}}\left[1\left\{\omega_{k} \neq \infty\right\}\left(\lambda\left(v_{k-1}(t)-v_{k}(t)\right)+e^{-\omega_{k}}-e^{-\omega_{k+1}}\right)\right]=0
$$

The line of reasoning employed in establishing Lemma 4.1 and Theorem 4.2 readily applies to $\mathbf{v}(\cdot)$ and $\pi_{\mathbf{v}(\cdot)}$ here, yielding that

$$
\pi_{\mathbf{v}(t)}\left(\omega_{k} \in \mathbb{R}_{+} \text {and } \omega_{k+1}=0\right)=1 \quad \text { if } v_{k}(t)=0
$$

and that $v_{1}(t)=0$ for $t>K(0) /(1-\lambda)$. In turn for such $t, b_{1}(t)=O(1)$ and $b_{2}(t)=o(1)$ as $n \rightarrow \infty$. The maximum queue size in equilibrium therefore tends to one, but the number of queues at that occupancy is substantially larger than the same number under the LCQ policy. In particular for large enough values of $t$ the total system occupancy $\sum_{k \geq 1} m_{k}(t)=\left(n / d_{n}\right) \sum_{k \geq 1} b_{k}(t)$ is $O\left(n / d_{n}\right)$. 


\section{References}

[1] M. Alanyali, "Asymptotically exact analysis of a loss network with channel continuity," The Annals of Applied Probability, vol. 13, no. 4, pp. 1474-1493, 2003.

[2] S. N. Ethier and T. G. Kurtz, Markov processes: Characterization and convergence, Wiley, 1986.

[3] A. Ganti, E. Modiano, and J. N. Tsitsiklis, "Optimal transmission scheduling in symmetric communication models with intermittent connectivity," IEEE Transactions on Information Theory, vol. 53, no. 3, pp. 998-1008, 2007.

[4] P. J. Hunt and T. G. Kurtz, "Large loss networks," Stochastic Processes and Their Applications, vol. 53, pp. 363-378, 1994.

[5] T. G. Kurtz, "Averaging for martingale problems and stochastic approximation," Lecture Notes on Control and Information Sciences, vol. 117, pp. 186-209, Springer-Verlag, 1992.

[6] M. J. Neely, "Order optimal delay for opportunistic scheduling in multi-user wireless uplinks and downlinks," 44th Allerton Conference on Communication, Control, and Computing, 2006.

[7] S. Shakkottai, R. Srikant, and A. L. Stolyar, "Pathwise optimality of the exponential scheduling rule for wireless channels," Advances in Applied Probability, vol. 36, no. 4, pp. 1021-1045, 2004.

[8] S. Shakkottai, "Effective capacity and QoS for wireless scheduling," IEEE Transactions on Automatic Control, vol. 53, no. 3, pp. 749-761, 2008.

[9] A. L. Stolyar, "Maxweight scheduling in a generalized switch: State space collapse and workload minimization in heavy traffic," The Annals of Applied Probability, vol. 14, no. 1, pp. 1-53, 2004.

[10] L. Tassiulas and A. Ephremides, "Dynamic server allocation to parallel queues with randomly varying connectivity," in IEEE Transactions on Information Theory, vol. 39, no. 2, 1993.

[11] N. D. Vvedenskaya, R. L. Dobrushin, and F. I. Karpelevich, "Queueing system with selection of the shortest of two queues: an asymptotic approach," in Problems of Information Transmission, vol. 32 , no. 1, pp. 15-27, 1996.

[12] L. Ying, R. Srikant, A. Eryilmaz and G. Dullerud, "A large deviations analysis of scheduling in wireless networks," IEEE Transactions on Information Theory, vol. 52, no. 11, pp. 5088-5098, 2006 . 
[13] S. Zachary and I. Ziedins, "A refinement of the Hunt-Kurtz theory of large loss networks with an application to virtual partitioning," The Annals of Applied Probability, vol. 12, pp. 1-22, 2002. 